\title{
Compact planar lenses based on a pinhole and an array of single mode metallic slits
}

\section{Q. Lévesque \\ P. Bouchon \\ patrick.bouchon@onera.fr \\ F. Pardo}

\section{J.-L. Pelouard}

\section{R. Haïdar}

\author{
Onera - The French Aerospace Lab, F-91761 Palaiseau, France \\ Laboratoire de Photonique et de Nanostructures (LPN-CNRS), F-91460 Marcoussis, France \\ Onera - The French Aerospace Lab, F-91761 Palaiseau, France
}

Laboratoire de Photonique et de Nanostructures (LPN-CNRS), F-91460 Marcoussis, France

Plasmonic lenses are based on complex combinations of nanoscale high aspect ratio slits. We show that their design can be greatly simplified, keeping similar performance while releasing technological constraints. The simplified system, called Huygens lens, consists in a central aperture surrounded by several identical single mode slits in a thin gold layer that does not rely anymore on surface plasmons. The focusing behaviour with respect to the position and number of slits is investigated, and we demonstrate the interest of this design to get compact array of lenses.

[DOI: http://dx.doi.org/10.2971/jeos.2013.13071]

Keywords: Nanolens, plasmonic, nanostructures, interferences, diffraction

\section{INTRODUCTION}

The development of integrated compact optical sensors requires the miniaturization of lenses at a mesoscopic scale[1]. However the downsizing of conventional dielectric-based optical devices leads to an increase of the curvature and the thickness of the object, thus giving rise to fabrication issues. Also, these lenses must be aligned and hold mechanically which results in a spatial limitation in the miniaturization of global optical system. In order to improve compactness and sturdiness of such systems, other alternatives implying planar lenses placed directly on top of the sensing device have been suggested [2]. In particular, plasmonic lenses (PL) have been extensively studied since 2005 [3]-[7]. In these lenses, phase delays allowing the constructive interference at the focal area are made of two terms. The first term stems from the transmission throughout the slit, which depends on the width of the slit. This is due to the effective refractive index associated to the guided mode inside the slits, which results from the coupling of surface plasmons propagating along the slit walls, and depends on its width $[3,8]$. The second term is driven by the position of the slit relative to the structuring axis according to the light path difference. Thus, an array of subwavelength slits of different widths in a planar metallic layer can be tailored to induce a lens-shaped phase modulation.

Getting a phase shift over the $[0-2 \pi]$ range requires narrow and high aspect ratio slits, but as the technological constraints determine the minimal slit width, the maximum avail- able phase shift is limited. Moreover, the index of the guided mode is highly sensitive to width variations, underlining the need to engrave these slits with high precision [8]. Therefore, the realization of such PL, which requires etching an array of heterogeneous high aspect ratio (defined as the ratio of height over width) slits while keeping nanometric precision, seems to be hardly achievable. Even for more realistic PL designs $[9,10]$, the tolerance on slit width remains a critical parameter. In contrast, other designs based also on plasmonics like nano-hole quasi-periodic array [11], or rather on diffraction effects like super-oscillatory lenses [12] or Fresnel zone plates, have been suggested. Fresnel zone plates are used either to focus in-plane surface plasmons $[13,14]$ or visible light [15]-[17]. However, they have always been demonstrated with a high number of zones, which could be a drawback against the miniaturization of systems and their integration in detector arrays.

In this letter, we show that the combination of a central aperture engraved in a thin metallic layer, and surrounded by several identical single mode slits leads to similar light focusing as in PL while dramatically releasing the technological constraints. Moreover, we show that a 3-slits structure allows to reach numerical aperture in the range of 1 to 3 while keeping a nearly constant efficiency, which is a step toward more compact devices. We call this system a Huygens lens (HL). The influence of the number of lateral slits as well as their position 

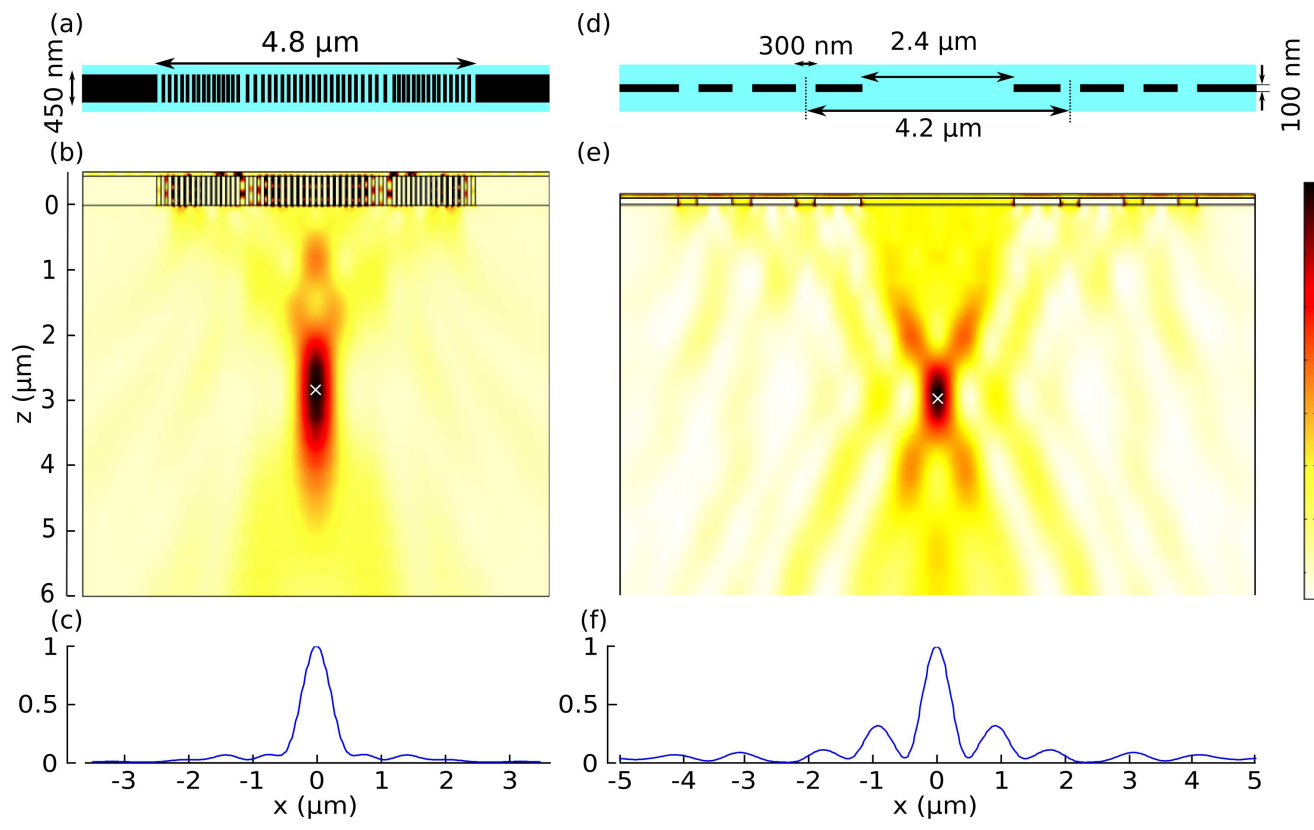

(e)

(g)
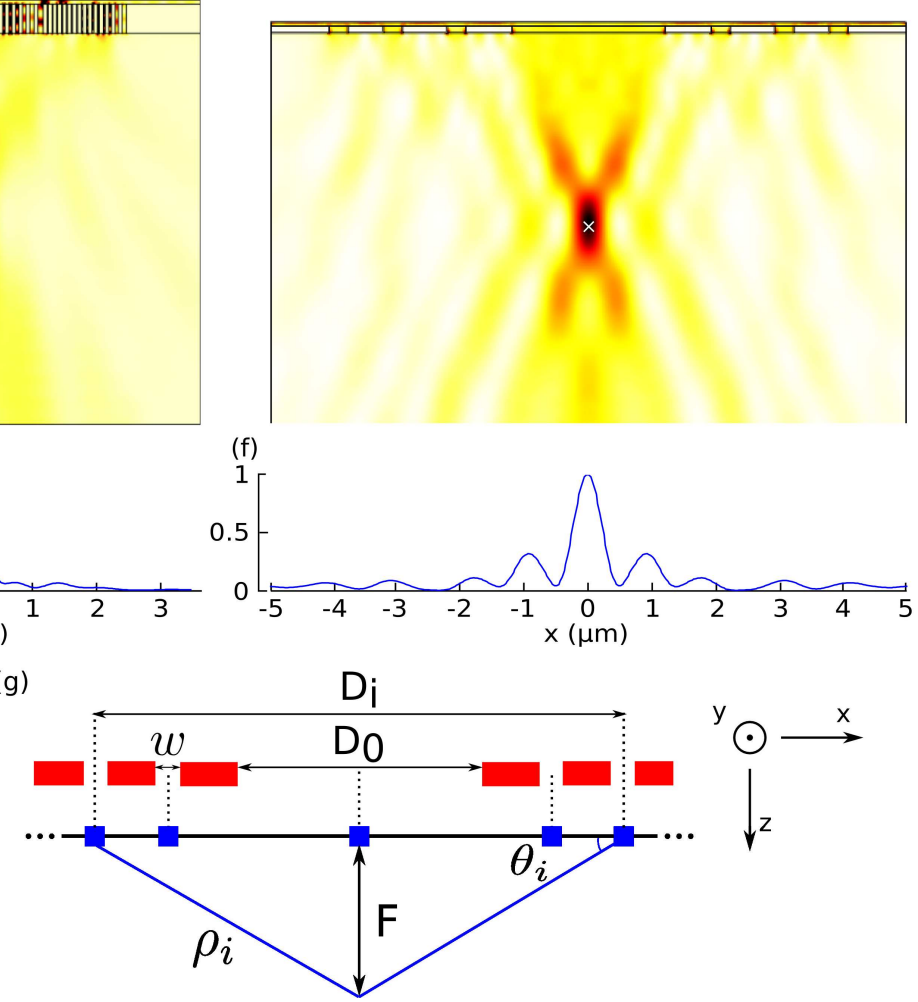

FIG. 1 Design for $3 \mu \mathrm{m}$ focal length of the (a) previously PL based on phase gradient [18] and (d) the HL structure based on central aperture surrounded by single mode slits. The thickness of the gold layer is respectively 450 and $100 \mathrm{~nm}$ surrounded by air. (b,e) Simulated electric field intensity map and (c,f) the related transverse profile at the focal point respectively for structure shown in (a) and (d). (g) Layout of the structure and the relevant geometric parameters.

relative to the central aperture on the focusing HL properties are investigated.

\section{HUYGENS LENS DESCRIPTION}

We consider a 2D-system, made of rectangular apertures engraved in a metallic layer along the x-direction, as represented in Figure 1(g). First, the width of the central aperture defines the focal length $F$. The solution of the Helmholtz equation in the central aperture is the Hankel function $H_{0}^{(1)}$ [19]. Then, the Rayleigh-Sommerfield integral of Hankel function for a rectangular aperture of width $D_{0}$ along x leads to:

$$
F=\frac{D_{0}^{2}}{3 \lambda}
$$

Let us emphasize that this formula holds for a cylindrical configuration and has been confirmed by exact rigorous electromagnetic computations. It slightly differs from the spherical case [18]. Secondly, several pairs of adjacent slits are added, in order to produce constructive interferences at the desired focal point. This addition also increases the lens aperture, thus improving the sharpness and the contrast of the focal spot. Considering the case of a symmetrical cylindrical lens under normal incidence illumination, the distance $D_{i}$ between the two slits forming the $i^{\text {th }}$ pair as drawn in Figure 1(g) must be set in order to have constructive interferences between the light exiting from the slits and the central aperture at the de- sired focal $F$. Therefore, the phase shift $\Delta \Phi$ must be:

$$
\Delta \phi=\frac{2 \pi}{\lambda}\left(F-\rho_{i}\right)=2 N \pi
$$

where $\rho_{i}$ is the distance between the $i^{t h}$ slits and the focal spot and $\mathrm{N}$ is a positive integer. Thus $D_{i}$ can be expressed as:

$$
D_{i}=2(F+N \lambda) \cos \left(\arcsin \left(\frac{F}{F+N \lambda}\right)\right)
$$

The HL design consists in an identical internal phase delay term for the central aperture and all slits, and it is no longer based on the phase modulation due to plasmonic behaviors. The gold is used only as an opaque optical medium wherein slits are engraved, thus only needing a gold layer thickness of a few skin depths ( $100 \mathrm{~nm}$ in the visible domain).

As reference, we choose the PL designed by Yu et al. represented in Figure 1(a) [18], which focuses a normal incident transverse magnetic (TM) plane wave at $\lambda=650 \mathrm{~nm}$ at a focal length of $3 \mu \mathrm{m}$. The electric field intensity map and its transverse profile at the focal point are shown in Figure 1(b) and $1(\mathrm{c})$. The simulations were performed with COMSOL software [20], using $\epsilon_{m}=-12.9+1.2 i$ for the gold permittivity [21] and the scattering boundaries conditions. As we can see, the focal spot is sharp and well contrasted. The thickness of the gold layer is fixed at $450 \mathrm{~nm}$, thus the phase delays are controlled by varying both the width and the position of the slits. This process leads to a 53-slit structure with aspect ratios 
of up to 37 which are hardly achievable. A much simpler HL was designed with the same focal length as shown in Figure $1(\mathrm{~d})$. It consists in a $2.8 \mu \mathrm{m}$ central slit engraved in a $100 \mathrm{~nm}$ gold layer, surrounded by 3 pairs of $300 \mathrm{~nm}$ wide slits filled with air. The corresponding electric field intensity map and its transverse profile at the focal point are plotted in Figures $1(\mathrm{e})$ and $1(\mathrm{f})$. Approximately $52 \%$ of the energy incident on the lens aperture is transmitted in the TM polarisation state, which corresponds to the geometrical aperture of the HL. It is comparable to the transmission of $67 \%$ obtained for the plasmonic lens. Comparison with Figures 1(b) and 1(c) points out similarities in the focusing behaviour. The focal length and spot area have closely the same value and shape respectively, however contrast is slightly lower in the HL design. Thus with a much simpler and realistic design, HL presents focusing properties comparable to those of a PL.

\section{INFLUENCE OF $D_{1}$ ON LIGHT FOCUSING}

The electric field intensity map is plotted in Figure 2(a) for a single aperture of width $D_{0}=3.2 \mu \mathrm{m}$, with an incident TM-polarized light at $\lambda=650 \mathrm{~nm}$. It produces a diffraction spot with a large depth of field, in the z-range of 4 to $8 \mu \mathrm{m}$ defined as the distance where the intensity value is above $90 \%$ of the maximum intensity. Inside this spot the phase value is nearly constant and appears to be a free parameter. The position of single mode slits fixes it and therefore influences the focal length of HL. Indeed in Figures 2(b)-(e), the electric field intensity map is plotted when one pair of external single mode slits is added to the aperture $D_{0}$, for various values of $D_{1}$ (resp. 4.2, 4.8, 5.4 and $6 \mu \mathrm{m}$ ). The addition of one pair of single mode slits appears to both increase the aperture of lens and reduce the depth of field of focal spot, defined by the longitudinal full width at half maximum (FWHM) of the electric field intensity $|E|^{2}$. It allows to select a specific focal point inside the range given by the central aperture depending on the value of $D_{1}$. Moreover, as can be seen, the intensity at the focal spot remains at the same level when $D_{1}$ is changed. The ability to tune the focal length at a micrometrical scale by only changing $D_{1}$ demonstrates the robustness of the design relatively to the position of external slits. These results still hold when considering other values of $D_{0}$. Indeed, the Figure 2(f) shows the focal length ranges obtained for $D_{0}$ values between $\lambda / 2$ and $15 \lambda$. For each value of $D_{0}, D_{1}$ is tuned from $D_{0}+w$ to $2 D_{0}$. The inset of Figure 2(f) shows the focusing for a HL with $D_{0}=\frac{3}{2} \lambda$ and $D_{1}=3 \lambda$. There is still a well defined focal spot close to the diffraction limit.

\section{INFLUENCE OF THE NUMBER OF EXTERNAL SLITS}

These results remain valid for a HL with additional external slits, and we now investigate how the focusing behavior of HL can be further improved in this case. Three to seven slits structures are considered, with the distances $D_{i}$ calculated fol-
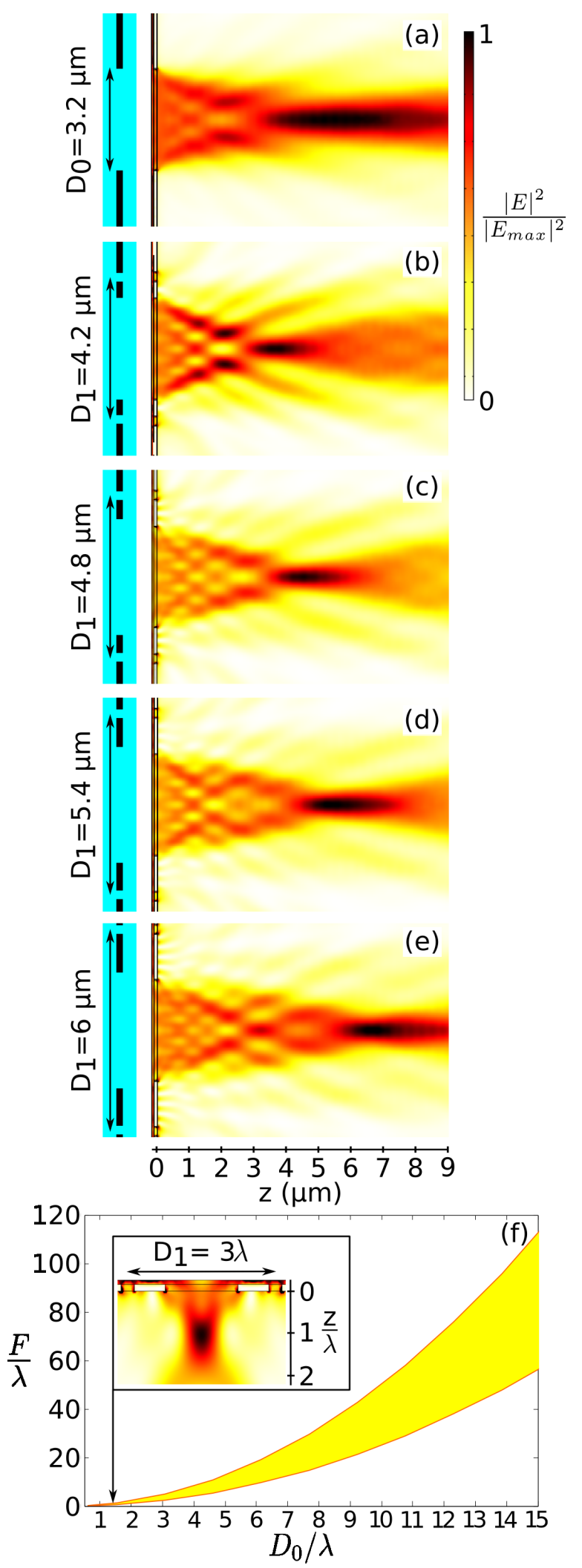

FIC. 2 (a) Electric field intensity map at $\lambda=650 \mathrm{~nm}$ for a central aperture alone, having a fixed width of $D_{0}=3.2 \mu \mathrm{m}$ and a gold thickness of $100 \mathrm{~nm}$. (b-e) Electric field intensity map of the previous central aperture surrounded by one pair of external $300 \mathrm{~nm}$ wide slits for respectively 4.2, 4.8, 5.4 and $6 \mu \mathrm{m}$ external diameter $D_{1}$ value. (f) The yellow area gives the focal length ranges attainable as a function of the central aperture width $D_{0}$, with $D_{1}$ values taken in a range from $D_{0}+w$ to $2 D_{0}$. The inset shows the electric field intensity map for a $\mathrm{HL}$ with $D_{0}=\frac{3}{2} \lambda$ and $D_{1}=3 \lambda$.

lowing Eq. (3) in order to get a $5 \mu \mathrm{m}$ focal length HL, as illustrated on the left of Figures 3(a)-(c). The focal spot area is 


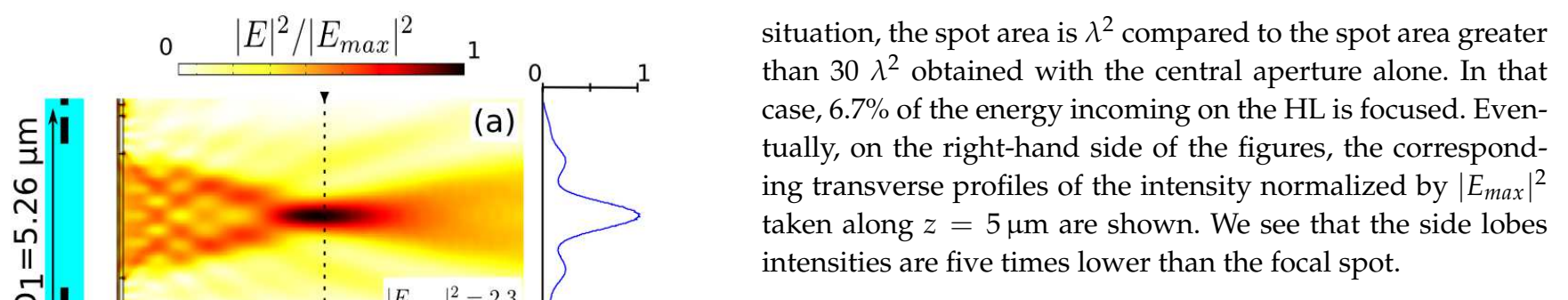

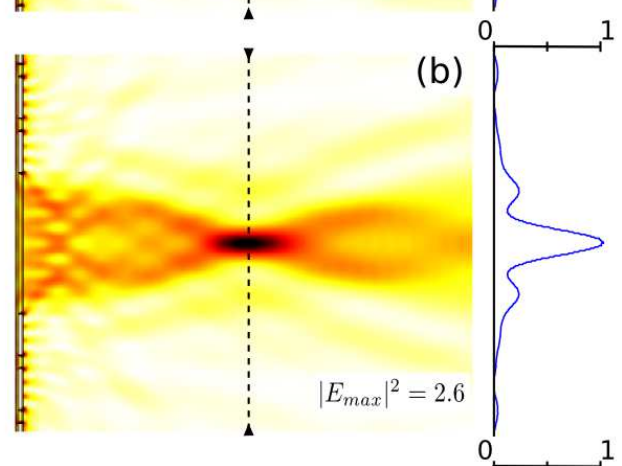
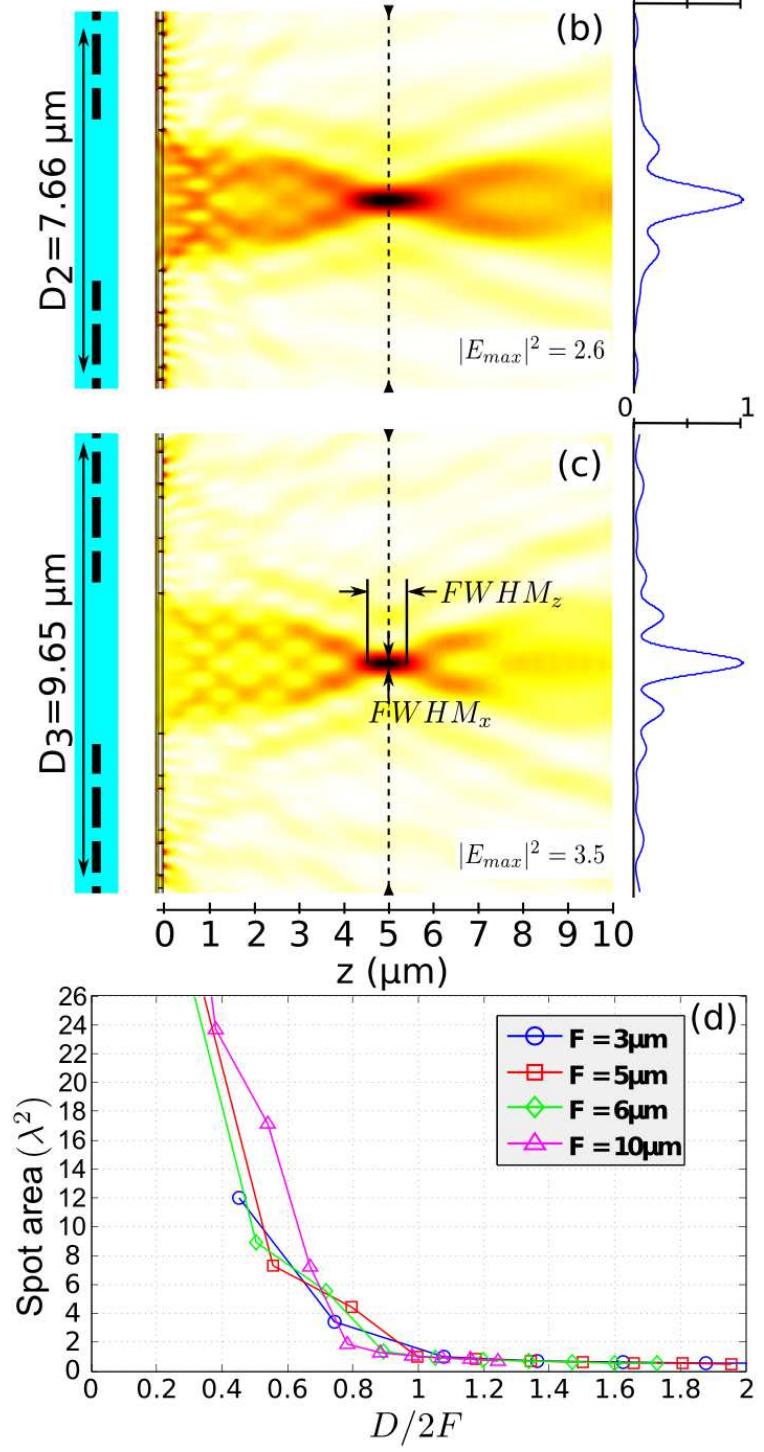

FIG. 3 (a-c) Electric field intensity map at $\lambda=650 \mathrm{~nm}$ for $\mathrm{HL}$ with respectively 3,5 and 7 -slits structure in a $100 \mathrm{~nm}$ thick gold layer illuminated by an unitary incident intensity. On the left are shown the considered structures. On the right, the corresponding transverse profiles of the intensity normalized by $E_{\max }^{2}$ taken along $z=5 \mu \mathrm{m}$ are shown. (d) Spot area as a function of the lens aperture $D$ normalized by $2 F$ for various values of $F$.

calculated from the transversal and longitudinal FWHM considering the approximation of an ellipsoidal shape. The quantitative improvement of the focusing figures is shown in the electric field intensity map in Figures 3(a)-(c). The larger the lens aperture, the smaller the focal spot area. Figure 3(d) illustrates the evolution of the spot area as a function of the normalized lens aperture $D / 2 F$ for various focal length values. It clearly shows that the enhancement stops when the number of slits leads to a total aperture larger than $2 F$. For this optimum

\section{PERIODIC ARRAY OF HUYGENS LENSES}

The identical single mode slits surrounding the central aperture in HL design could be used to improve the compactness of an array of lenses. In order to illustrate this, the intensity map of a periodic 3-slits HL (Figure 4(a)) has been plotted in Figure 4(b) on three periods. The dimensions of the HL are the same as the aperiodic 3-slits HL shown in Figure 3(a) repeated with a period $\Lambda=6.46 \mu \mathrm{m}$ (Floquet periodic conditions have been implemented in COMSOL). The periodic HL has roughly the same focusing behavior as the aperiodic one, but the focal length is slightly shifted to $F=5.3 \mu \mathrm{m}$ and the spot area is smaller. The transverse profile of the normalized electric field is plotted at the focal length in the periodic (red continuous curve) and aperiodic (green dashed curve) cases in Figure 4(c). The focal spot has a higher intensity and a smaller transversal FWHM in the periodic case. In fact, in the periodic lens, the slits of the neighboring lenses also contribute to the focusing. This means that a $(2 N+1)$-slits periodic lens has a similar behavior to a $(4 N+1)$-slits aperiodic HL, where $N$ can be a half integer. The transverse profile of the 5-slits aperiodic HL is also plotted in 4(c) (blue dashed curve), and confirms that the peak intensity and FWHM are very close to the 3 -slits periodic case. In summary, the periodicity of HL allows to reduce the aperture needed to achieve given focusing performances.

\section{CONCLUSION}

We have proposed the HL design that consists in an identical internal phase delay term for all slits. Therefore very narrow slits are no longer needed to produce high phase modulations. These outcomes strongly release the technological constraints, in particular aspect ratios of designed HL can be very low (typically $\sim 1$ ) and keep good focusing properties. The HL represents an alternative design to PL in order to make planar lens with a more achievable fabrication. HL with a fixed or variable focal length can be designed following a set of simple analytical equations. Furthermore, an asymmetric distribution of the slits can also be considered. This study paves the way to the conception of structures with more complex functionalities while keeping a viable technology.

\section{ACKNOWLEDGEMENTS}

The authors thank Benjamin Portier for fruitful discussions. QL acknowledges financial support from the Direction Générale de l'Armenent. 


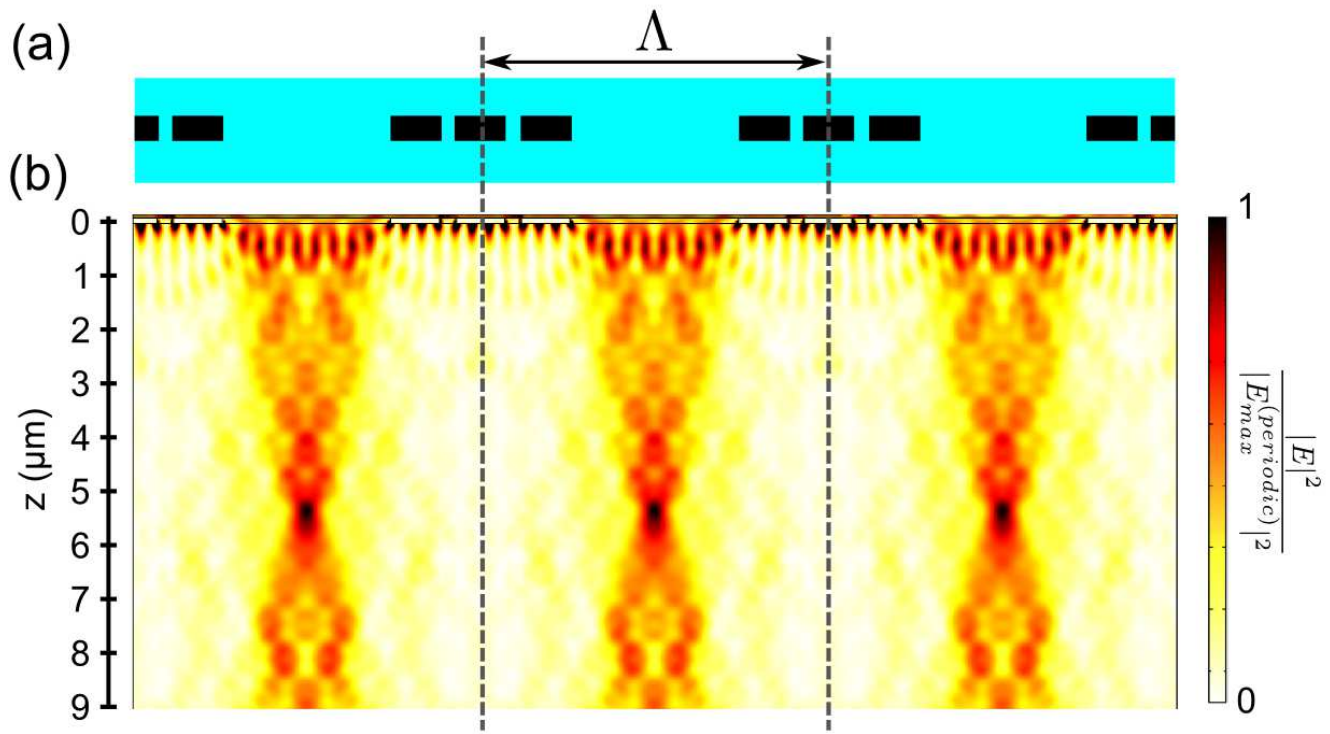

(c)

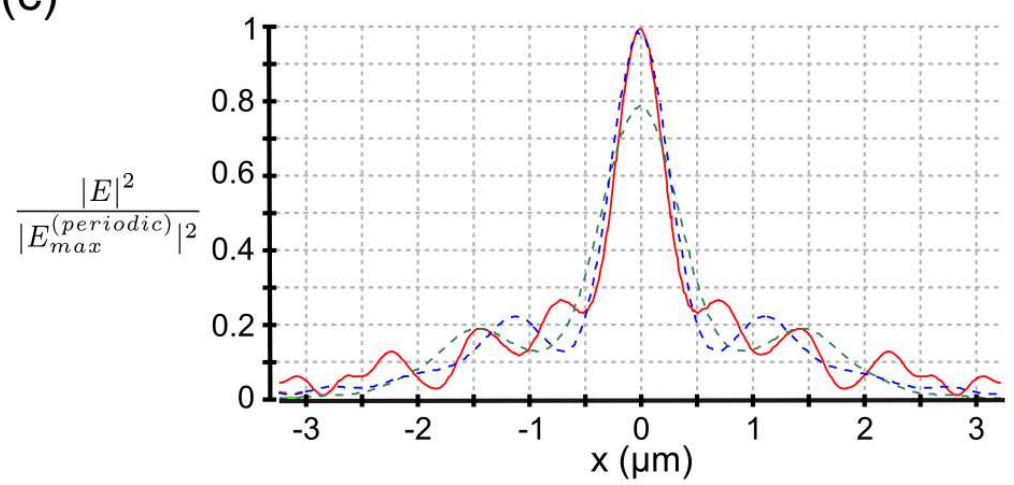

FIG. 4 (a) Periodic 3-slits HL design, with a period of $\Lambda=6.46 \mu \mathrm{m}$. (b) Normalized electric field intensity map of the previous design at $\lambda=650 \mathrm{~nm}$ shown on three periods. (c) Transverse profile of the intensity normalized by the maximum intensity in the periodic case taken at the focal point for the periodic array (red continuous curve) compared with the transverse profiles obtained with aperiodic 3-slits $\mathrm{HL}$ (green dashed curve) and 5 -slits $\mathrm{HL}$ (blue dashed curve).

\section{References}

[1] E. Ozbay, "Plasmonics: merging photonics and electronics at nanoscale dimensions," Science 311, 189-193 (2006).

[2] L. Verslegers, P. Catrysse, Z. Yu, J. White, E. Barnard, M. Brongersma, and S. Fan, "Planar lenses based on nanoscale slit arrays in a metallic film," Nano Lett. 9, 235-238 (2008).

[3] H. Shi, C. Wang, C. Du, X. Luo, X. Dong, and H. Gao, "Beam manipulating by metallic nano-slits with variant widths," Opt. Express 13, 6815-6820 (2005).

[4] L. Verslegers, P. Catrysse, Z. Yu, and S. Fan, "Planar metallic nanoscale slit lenses for angle compensation," Appl. Phys. Lett. 95, 071112-071112 (2009).

[5] L. Verslegers, P. Catrysse, Z. Yu, and S. Fan, “Deep-subwavelength focusing and steering of light in an aperiodic metallic waveguide array," Phys. Rev. Lett. 103, 33902 (2009).

[6] Y. Fu, and X. Zhou, "Plasmonic lenses: a review," Plasmonics 5, 287-310 (2010)

[7] S. Ishii, V. M. Shalaev, and A. V. Kildishev, "Holey-metal lenses: sieving single modes with proper phases," Nano Lett. 13, 159-163 (2012).

[8] S. Collin, F. Pardo, and J. Pelouard, "Waveguiding in nanoscale metallic apertures," Opt. Express 15, 4310-4320 (2007).
[9] Q. Chen, and D. Cumming, "Visible light focusing demonstrated by plasmonic lenses based on nano-slits in an aluminum film," opt. Express 18, 14788-14793 (2010).

[10] Q. Chen, "Effect of the number of zones in a one-dimensional plasmonic zone plate lens: simulation and experiment," Plasmonics 6, 75-82 (2011).

[11] F. M. Huang, N. Zheludev, Y. Chen, and F. Javier Garcia de Abajo, "Focusing of light by a nanohole array," Appl. Phys. Lett. 90, 091119-091119 (2007).

[12] E. T. Rogers, S. Savo, J. Lindberg, T. Roy, M. R. Dennis, and N. I. Zheludev, "Super-oscillatory optical needle," Appl. Phys. Lett. 102, 031108-031108 (2013).

[13] L. Feng, K. A. Tetz, B. Slutsky, V. Lomakin, and Y. Fainman, “Fourier plasmonics: Diffractive focusing of in-plane surface plasmon polariton waves," Appl. Phys. Lett. 91, 081101-081101 (2007).

[14] J. Wang, X. Wu, and J. Zhang, "Imaging properties of Fresnel zone plate-like surface plasmon polariton launching lenses," Opt. Express $18,6686-6692$ (2010).

[15] F. Hao, R. Wang, and J. Wang, "Design and characterization of a micron-focusing plasmonic device," Opt. Express 18, 15741-15746 (2010).

[16] R. Mote, S. Yu, B. Ng, W. Zhou, and S. Lau, "Near-field focusing properties of zone plates in visible regime-New insights," opt. Express 16, 9554-9564 (2008). 
[17] H. Ye, C.-W. Qiu, K. Huang, J. Teng, B. Lukýanchuk, and S. P. Yeo, "Creation of a longitudinally polarized subwavelength hotspot with an ultra-thin planar lens: vectorial Rayleigh-Sommerfeld method," Laser Phys. Lett. 10, 065004 (2013).

[18] Y. Yu, and H. Zappe, "Effect of lens size on the focusing performance of plasmonic lenses and suggestions for the design," 0 pt. Express 19, 9434-9444 (2011).
[19] M. Abramowitz, and I. A. Stegun, Handbook of Mathematical Functions: With Formulars, Graphs, and Mathematical Tables (Dover Publications, Mineola, 1964).

[20] Comsol Multiphysics 4.2 http://www.comsol.com.

[21] P. Johnson, and R. Christy, "Optical constants of the noble metals," Phys. Rev. B 6, 4370 (1972). 